Théologiques

Théologiques

\title{
La lettre d'amour comme genre théologique
}

\section{François Nault}

Volume 8, numéro 1, printemps 2000

La superstition

URI : https://id.erudit.org/iderudit/005020ar

DOI : https://doi.org/10.7202/005020ar

Aller au sommaire du numéro

\section{Éditeur(s)}

Faculté de théologie de l'Université de Montréal

\section{ISSN}

1188-7109 (imprimé)

1492-1413 (numérique)

Découvrir la revue

\section{Citer cet article}

Nault, F. (2000). La lettre d'amour comme genre théologique. Théologiques, 8(1), 105-124. https://doi.org/10.7202/005020ar

\section{Résumé de l'article}

Rapporter le geste théologique à la lettre d'amour, c'est s'inscrire d'entrée dejeu dans un espace assez inconfortable. C'est s'engager à s'exprimer en exilédans l'interstice formé par un double écart : d'une part, l'écart qui séparel'écriture théologique de l'écriture amoureuse et, d'autre part, l'écart quiéloigne chacune de ces écritures de son lieu de circulation habituel. Loin devouloir résorber ces écarts, je voudrais m’y enfoncer comme il faut, enétendre les ressorts afin de mesurer le jeu qu'ils permettent. Pour ce faire, jepropose de prendre comme fil conducteur La carte postale de JacquesDerrida et l'horizon de questionnement que ce livre étrange délimite. 


\title{
La lettre d'amour comme genre théologique ${ }^{\star}$
}

François NAULT

Université Saint-Paul

Ottawa

\begin{abstract}
"Parlons-nous de la même chose lorsque nous parlons d'amour? Et de laquelle? L'épreuve amoureuse est une mise à l'épreuve du langage : de son univocité, de son pouvoir référentiel et communicatif. »

J. Kristeva, Histoires d'amour ${ }^{1}$
\end{abstract}

Rapporter le geste théologique à la lettre d'amour, c'est s'inscrire d'entrée de jeu dans un espace assez inconfortable. C'est s'engager à s'exprimer en exilé, dans l'interstice formé par un double écart : d'une part, l'écart qui sépare l'écriture théologique de l'écriture amoureuse et, d'autre part, l'écart qui éloigne chacune de ces écritures de son lieu de circulation habituel.

Le premier écart est formé par toutes les résistances qu'on peut opposer à l'association du théologique au genre épistolaire. Comprendre l'écriture théologique à partir de la lettre - de la lettre d'amour - équivaudrait à une remise en cause des valeurs de vérité, de sérieux, de réflexivité, de rationalité, voire d'objectivité, sans lesquelles la théologie ne serait plus elle-même mais pure poésie, pure rhétorique ou pure passion. Comprendre l'écriture théologique à partir de la lettre d'amour équivaudrait à la rattacher à l'impétuosité d'un geste, à l'éclatement (du moins potentiel) de ses formes canoniques, à l'emportement d'un sujet ne s'appartenant plus et surtout, plus gra-

\footnotetext{
* Texte remanié d'une conférence prononcée à l'Université Saint-Paul (Ottawa), le 26 mars 1999.

1. J. KRISTEVA, Histoires d'amour, Paris, Denoël, (Coll. " Folio/essais ", 24), 1983, p. 10.
} 
vement, à la dissolution de son objet. La lettre d'amour porterait en elle trop d'espoir fragile (c'est-à-dire de désespoir) pour croire en l'amour et en l'Autre, pour s'y appuyer : le réseau métaphorique qui se tisse autour de l'écriture amoureuse ne pourrait qu'incliner à l'athéisme.

Le second écart dans lequel se loge le discours que je cherche à tenir est formé par toutes les résistances qu'on peut opposer à l'expulsion de l'écriture théologique et de l'écriture amoureuse de leur champ opérationnel respectif. Comprendre l'écriture théologique à partir de la lettre d'amour tendrait à ramener la délibération théologique dans l'espace clos d'une correspondance privée et secrète : la théologie comme prière. À l'inverse, la sollicitation théologique de la lettre d'amour projetterait cette dernière hors du cercle intime formé par son destinataire et son destinateur : la lettre d'amour comme journal public.

Loin de vouloir résorber les écarts que je viens d'évoquer sommairement - écarts qui étaient d'ailleurs déjà suggérés par l'intitulé - , je voudrais m'y enfoncer comme il faut, en étendre les ressorts afin de mesurer le jeu qu'ils permettent. Pour ce faire, je propose de prendre comme fil conducteur La carte postale de Jacques Derrida et l'horizon de questionnement que ce livre étrange délimite. ${ }^{2}$ Il ne faudra pas se méprendre cependant sur la facture apparemment objective du discours que je tiendrai : " commenter un texte, c'est comme faire une analyse ", disait Lacan ; ${ }^{3}$ c'est une façon de mettre le sujet en marche. Le « je » s'immisce et travaille davantage dans la neutralité du « nous» qu'il ne s'y perd...

Ainsi il faudra être attentif aux anfractuosités multiples d'un discours - le mien - se posant comme " analyse " ou "discours objectif » sur l'amour. Ce que je cherche à suggérer ici et qui ne sera en aucun cas énoncé formellement dans le corps du texte (c'est-à-dire tout ce qui exclut les présentes remarques liminaires et l'apostrophe finale), c'est la possibilité que la sécheresse descriptive de ma lecture ne serve au fond qu'à dissimuler la la passion amoureuse qui la nourrit.

2. J. DERRIDA, La carte postale : de Socrate à Freud et au-delà, Paris, Flammarion, (Coll. " La philosophie en effet »), 1980.

3. J. LACAN, Les écrits techniques de Freud: le séminaire Livre I, Paris, Seuil, 1975, p. 87. 
Cette équivoque fondamentale ne sera pas résolue — l'entre-deux restera non dialectisable - car la lettre d'amour, si c'en est une, doit forcément se poser dans une "privatisation absolue " : ${ }^{4}$ " Je t'aime cela ne se publie pas. ${ }^{5}$ On ne pourra donc jamais décider avec certitude si le discours que je tiens relève d'une vague aspiration à l'écriture amoureuse ou s'il en constitue déjà une forme secrète d'achèvement.

Plus encore, on ne saura jamais vraiment à qui cette lettre - si c'en est une - se destine : l'autre dont il sera question (le ou la destinataire), est-ce une femme (réelle ou imaginaire) ou encore Dieu? D'ailleurs, y a-t-il forcément une seule adresse? D'un destinataire à l'autre, de l'autre à l'Autre, que se passe-t-il? Plus directement encore, je pose la question : la théologie ne serait-elle pas (encore) théologique lorsque, cessant de dire «Dieu » (le mot « Dieu »), elle devient amoureuse et s'adresse à Lui — la théologie comme « jeu d'adresse(s) » ? 6

«Ce qui est caressé n'est pas touché à proprement parler. Ce n'est pas la velouté ou la tiédeur de cette main donnée dans le contact que cherche la caresse. C'est cette recherche de la caresse qui en constitue l'essence, par le fait que la caresse ne sait pas ce qu'elle cherche. Ce "ne pas savoir", ce désordonné fondamental en est l'essence. »

E. Lévinas, Le temps et l'autre ${ }^{7}$

4. La carte postale, p. 157.

5. Ibid., p. 263.

6. «Un terme, lui-même ambigu, pourrait définir ces procédures qui détachent la langue de son fonctionnement naturel pour la modeler sur les passions de sujets locuteurs : l'adresse, car d'une part la destination du discours l'emporte sur la validité de l'énoncé (il s'agit de paroles “adressées”, sans qu'on puisse savoir finalement par qui et à qui), et d'autre part une dextérité vise à éveiller dans les mots, par leurs corrélations avec d'autres, les possibilités que tenait en sommeil la domination de la chose signifiée [...]. Jeu d'adresse(s), donc. ", M. de CERTEAU, La fable mystique, Paris, Gallimard, (Coll. "Bibliothèque des histoires "), 1982, p. 195.

7. E. LÉVINAS, Le temps et l'autre, Montpellier, Fata Morgana, 1979, p. 82 
À qui s'adresse la lettre? De qui vient-elle? Que contient-elle? Ces questions ouvrent La carte postale sans toutefois résumer ou rassembler ce qui s'y dessine. En ouverture, avant même de procéder aux « envois ", Derrida demande : "Qui écrit! À qui? Et pour envoyer, destiner, expédier quoi? À quelle adresse? "Questions immenses sans réponse possible : "Sans aucun désir de surprendre, et par là de capter l'attention à force d'obscurité, je dois à ce qui me reste d'honnêteté de dire que finalement je ne le sais pas. " ${ }^{8}$ Derrida se réfugie ainsi dans un aveu de non-savoir — refusant de répondre aux questions qu'il soulève - , tout en reconnaissant le caractère pénible et douloureux de l'indétermination entourant la lettre et son envoi :

Que les signataires et les destinaires ne soient pas toujours visiblement et nécessairement identiques d'un envoi à l'autre, que les signataires ne se confondent pas forcément avec les envoyeurs ni les destinataires avec les récepteurs, voire avec les lecteurs (toi par exemple), etc., vous en ferez l'expérience et le sentirez parfois très vivement, quoique confusément. C'est là un sentiment désagréable que je prie chaque lecteur, chaque lectrice de me pardonner. ${ }^{9}$

Mais la question de la lettre ne mériterait pas d'être promue au rang de "problème " — me semble-t-il — si elle ne renvoyait qu'à un "sentiment désagréable "; en fait, ce sentiment, écrit Derrida, "vous met en rapport, sans discrétion, avec de la tragédie. ${ }^{10}$ Le problème de la lettre, ou plus précisément de l'envoi de la lettre, est tragique pour autant que l'envoi ne garantit pas la relation — le lien — qu'il désire désespérement instituer : "La "liaison" [...] (amoureuse ou postale), voilà le mot. » ${ }^{11}$ Le drame vient — sans qu'on l'appelle — de l'absence de lien ${ }^{12}$. Mais surtout, le drame provient du caractère non

8. La carte postale, p. 9.

9. Ibidem.

10. Ibidem.

11. Ibid., p. 124.

12. "Quand aucun fil ou sans-fil ne me relie à rien, à toi, je meurs d'angoisse. ", ibid., p. 33. Il serait sans doute intéressant d'expliciter le rapport entre la thématique du fil (téléphonique) et celle du " oui » — pour cela, il faudrait relire Ulysse gramophone: "(Allô, oui : j'écoute, j'entends que tu es là, prêt à parler au moment où je suis prêt à parler avec toi). Au commencement le téléphone, oui, au commencement du coup de téléphone. ", J. DERRIDA, Ulysse gramophone : deux mots pour Joyce, Paris, Galilée, (Coll. " La philosophie en effet »), 1987, p. 81. 
assuré du lien, de sa précarité, car " une lettre peut toujours ne pas arriver à destination. " ${ }^{13} \mathrm{Il}$ faut toujours craindre qu'une lettre se perde, que la correspondance soit rompue. ${ }^{14}$

À ne pas s'y tromper, la question qui nous occupe est religieuse de part en part : la lettre d'amour demeure impensable en dehors de la référence à la religion comme "lien" (religare) - qui unit ou rapproche - et comme "scrupule » (religio) - qui retient. ${ }^{15}$ Évidemment, cette double détermination de la religion (lien et scrupule) nous conduit à interpréter la « chose religieuse » dans son rapport à la « chose sexuelle », c'est-à-dire dans son rapport au «serment qui lie deux désirs $»{ }^{16}$

Manière de dire que le mot « religion » ne renvoie pas à un quelconque au-delà mais s'attache au corps (de la lettre); il surgit là même où les corps se rencontrent : "Le lit — comme une lettre ouverte. » ${ }^{17}$ On ne comprendra rien à la Carte postale de Derrida si on n'en fait pas une lecture érotique, si on ne la lit pas comme la trace d'une rencontre charnelle. Rencontre paradoxale et fragile puisque la destinataire des lettres ne cesse de se détourner - interdiction qu'on la

13. La carte postale, p. 135. Autre texte : «Une lettre n'arrive pas toujours à destination et, dès lors que cela appartient à sa structure, on peut dire qu'elle n'y arrive jamais vraiment, que quand elle arrive, son pouvoir-ne-pas-arriver la tourmente d'une dérive interne. ", ibid., p. 517. Voir aussi J. DERRIDA, "Comme si c'était possible, "within such limits"... ", Revue internationale de philosophie 205 (1998), 516-517.

14. "J'ai [...] l'impression, sans pouvoir préciser, qu'une lettre de moi s'est perdue. Anxiété juive! Au lieu de craindre que les lettres n'arrivent bien! », F. KAFKA, Lettres à Milena, trad. A. Vialatte et C. David, Paris, Gallimard, (Coll. "L'imaginaire », 200), 1988, p. 31.

15. Voir E. BENVENISTE, Le vocabulaire des institutions indoeuropéennes, Paris, Minuit, 1969, p. 269-270.

16. "Si tu meurs avant moi, je te garderai, si je meurs avant toi, tu me porteras en toi, l'un gardera l'autre, l'aura déjà gardé depuis la première déclaration. Cette double intériorisation ne serait possible ni dans l'intériorité monadique ni dans la logique de l'espace et du temps “objectifs”. Elle a pourtant lieu chaque fois que j'aime. ", J. DERRIDA, "L'aphorisme à contretemps ", dans Psyché : inventions de l'autre, Paris, Galilée, (Coll. " La philosophie en effet »), 1987, p. 524.

17. La carte postale, p. 40. 
regarde de face, comme Yahvé dans le Buisson ardent (Ex 3,1-6) : "La question se pose quand tu es dans la pièce à côté, voire quand dans la même pièce, te tournant à peine le dos, je t'écris encore, quand je laisse un mot sous ton oreiller ou dans la boîte aux lettres en partant, l'essentiel n'étant pas que tu sois absente ou présente au moment où je t'écris mais que je ne sois pas là, moi, quand tu lis. » 18

Dans cette scène d'écriture, il n'est pas surprenant que la figure d'Euridyce soit évoquée. ${ }^{19}$ Et avec elle, l'impossible transparence. Euridyce est la femme qui suit, dans l'ombre, sans qu'on puisse la voir ; il faut toujours la deviner, la chercher du regard mais sans se retourner, sans succomber à l'immédiateté : «Voudrais ne m'adresser, tout droit, directement, sans courrier, qu'à toi mais je n'y arrive pas et c'est le fond du malheur. Une tragédie, mon amour, de la destination. ${ }^{20}$ Une nouvelle fois, encore et toujours, la tragédie : la lettre nous y conduit fatalement.

"Accouplement des deux dans le même qui paralyse toute la scène. Sans espace entre lui et elle, eux et elles. »

L. Irigaray, La croyance même $e^{21}$

Le rêve d'une " adresse sans courrier » relève d'une aspiration à la proximité. L'adresse qui n'a pas besoin d'adresse - de marque identifiante - interpelle l'autre du plus proche : elle est contact. La lettre d'amour, s'écrivant au contraire dans la distance, implique toujours une certaine "différance » du contact ; la lettre d'amour n'est jamais sûre d'atteindre ce qu'elle vise, même si elle lui arrive : "Quand je t'appelle mon amour, mon amour, est-ce toi que j'appelle ou mon amour? Toi, mon amour, est-ce toi que je nomme ainsi, à toi que je m'adresse? Je ne sais pas si la question est bien formée, elle me fait peur. " ${ }^{22}$ Parce qu'elle s'inscrit toujours dans une langue, la lettre d'amour est traversée par l'ambiguïté ; elle s'écrit dans l'écart des

18. Ibid., p. 87.

19. Voir ibid., p. 25.

20. Ibid., p. 27.

21. L. IRIGARAY, La croyance même, Paris, Galilée, (Coll. "Débats »), 1983, p. 60.

22. La carte postale, p. 12. 
mots, dans l'ouverture infinie du sens. Si bien qu'il n'y aura jamais une seule adresse...

Pourtant la lettre d'amour est indissociable de la singularité d'un désir - désir d'atteindre, non pas une autre ou de l'autre, mais cette autre-ci : l'autre en tant qu'unique. La lettre est pour Milena ou pour Héloïse, pour Gala ou pour Brenda, ou encore pour une autre, pas n'importe laquelle - celle qui, même en n'étant pas nommée comme telle, se reconnaît pourtant dans un « toi » : «Une idée complètement folle : de n'écrire qu'à toi, à toi toute seule, à l'exclusion de tout autre destinataire possible. ${ }^{23}$ En tant que trace singulière et singularisante, la lettre d'amour cherche à instituer l'autre en tant qu'autre, en l'appelant par son nom... même quand elle ne la nomme pas. Il n'y aura qu'une adresse...

Mais si la lettre d'amour s'écrit toujours dans la distance, elle s'écrit également en opposition à la distance, contre elle; elle désire combler la béance et se résoudre en elle. Le véritable secret de la lettre d'amour - ce qui ne cesse de se nier en se disant et qui, par là, se dit en se niant - réside dans le désir d'être (dans) l'autre, littéralement : "Le secret de la lettre d'amour, c'est de croire à l'indistinction, à l'indifférenciation entre moi et toi, c'est qu'il n'y ait pas d'expéditeur, pas d'adresse ; c'est de bouleverser l'ordre de la communication, c'est d'être parole errante entre toi et moi, que nous pouvons nous approprier, l'un dans l'autre, l'un pour l'autre. ${ }^{24}$

L'écriture - lorsqu'elle est amoureuse - appelle l'autre (à soi et dans soi). Elle lui dit «viens! "; elle lui dit «viens » éternellement. ${ }^{25}$ Ce désir d'indistinction qui supporte la lettre d'amour est lisible

\section{Ibid., p. 181.}

24. D. BAJOMÉE, "La lettre d'amour ", Cahiers internationaux de symbolisme 62-63-64 (1989), p. 7. Le lien noue et scelle l'union : « Je connais le lien qui me lie à toi (tu fais partie de moi, même si je ne devais jamais te revoir). ", F. KAFKA, Lettres à Milena, p. 56-57.

25. Il faudrait évidemment mettre en oeuvre toute une série d'analyses couvrant les horizons multiples ouverts par la question du "viens ». Il faudrait établir les rapprochements qui s'imposent avec le «viens» de Maurice BLANCHOT dans L'arrêt de mort (Paris, Gallimard, 1948); il faudrait aussi lire La carte postale en dégageant la récurrence de ses motifs apocalyptiques, rattacher son « viens » au « viens » de l'Apocalypse de Jean 
jusqu'au plan formel. L'écriture amoureuse tend en effet à se concentrer en une seule expression, une " presque-phrase ", trois petits mots : "Qui que tu sois, mon amour, et même si tu trembles de n'en rien savoir toi-même, n'en doute plus : je n'ai jamais aimé que toi. Voilà déjà longtemps que tu ne peux plus me dire "je t'aime". Moi je le peux et cela suffit, ton amour est sauf. » ${ }^{26}$ " Je t'aime » : voilà, tout aura été dit - car rien n'a été dit. ${ }^{27}$ L'essentiel aura passé entre ces mots, dans leur profération magique ; dans cette expression - ou plutôt dans ce souffle $e^{28}$, l'appel à l'union des corps (en un seul corps) aura été bien compris. "Je t'aime. Je serai toi, tu seras moi. "

Par ailleurs, l'écriture du «viens! — précisément en tant qu'écriture - dit le désir de l'autre en l'inscrivant dans l'horizon du manque. La lettre d'amour dit toujours "viens! » dans un espace d'attente, dans un lieu où l'autre n'est pas, là où on attend (encore) sa venue : qu'il vienne, qu'il s'approche, plus près, qu'il cesse d'être autre - aussi bien dire qu'on attend l'impossible, Godot, Dieu. ${ }^{29}$ Puisque la venue de l'autre signerait la mort de l'autre comme autre, la mort de l'amour et la mort de l'écriture. Aussi, la lettre d'amour, tout en appelant l'autre et en allant à sa rencontre, veille à préserver la distance : "Je vais à ta rencontre, c'est tout ce que je sais en moi, mais aussi que je ne te rejoindrai jamais, que jamais tu ne m'arriveras :

(Ap 22, 20). Mais la référence biblique au «viens » déborde le contexte apocalyptique ; on la retrouve notamment dans le Cantique des cantiques : "Viens du Liban, ô fiancée, viens du Liban, fais ton entrée. [...] Tu me fais perdre le sens, ma soeur, ô fiancée, tu me fais perdre le sens par un seul de tes regards, par un anneau de ton collier! » (Ct 4,8-9).

26. La carte postale, p. 239.

27. "Passé le premier aveu, "je t'aime” ne veut plus rien dire, il ne fait que reprendre d'une façon énigmatique, tant elle paraît vide, l'ancien message. ", R. BARTHES, Fragments d'un discours amoureux, Paris, Seuil, 1977, p. 175. 28. «Un jour quand l'un de nous ne pourra plus dire "je t'aime", il suffira que l'autre encore en ait le souffle, rien n'aura changé. ", La carte postale, p. 195

29. "Attendre, l'autre... Qu'attend-on quand on attend l'autre? L'autre aimé, le "bien-aimé"? On "attend Godot"... On attend Dieu. [...] On attend Dieu, c'est-à-dire l'impossible. L'impossible "ajointement” de ce qui ne peut que demeurer "disjoint". », M.-L. MALLET, « Ombres d'Eurydice... », dans M. LISSE (dir.), Passions de la littérature, Paris, Galilée, 1996, (Coll. « La philosophie en effet »), p. 299. 
c'est cela, tu vois, qui déjà m'est arrivé et dont je ne peux que m'essouffler à tenir compte. ${ }^{30}$

De façon plus précise, la lettre d'amour travaille au profit de cette rencontre à venir (qui ne vient jamais) en suscitant un double mouvement : de soi à l'autre et de l'autre à soi. L'écriture amoureuse est l'inscription d'un geste en direction de l'autre (l'écriture comme exposition, comme don de soi) et une tentative d'attirer l'autre à soi (viens!). De ce point de vue, la lettre d'amour est traversée par un insatiable désir de séduction : "Qu'en mon absence tu sois encore séduite en ma confession jusqu'à mourir d'amour. Je ne fais rien en fin de compte qui ne s'intéresse à te séduire, à te détourner de toi pour te mettre en chemin vers moi, uniquement. ${ }^{31}$ (Il faut être attentif au caractère en quelque sorte absolu de la machination séductrice : « je ne fais rien qui ne s'intéresse à te séduire " — traduisons : " te séduire est tout ce qui m'intéresse ». La machination - je le souligne en passant - revêt ici un caractère en quelque sorte obsessif.)

L'apostrophe («viens!») constitue évidemment l'un des ressorts possibles de la machination. Mais l'écriture amoureuse est irréductible à cette rhétorique ; pour séduire l'autre, elle emprunte d'autres voies. La lettre d'amour paraît notamment indissociable d'une rhétorique de l'échec ou du discrédit. Il suffit de relire quelques Lettres à Milena pour s'en convaincre : " cette rage de lettres est insensée »; "pardonne-moi donc, Milena, mes lettres »; « je ne suis pas très satisfait de cette lettre »; "j'ai honte de mes lettres » ${ }^{32}$; etc. Dans les « envois » de La carte postale, Derrida sollicite également ce discours de l'amour qui se nie lui-même, qui appelle sa déflagration : "cette "correspondance" [...] nous a débordés, c'est pour ça qu'il aurait fallu tout brûler »; " ne plus écrire, la seule affirmation, la seule chance (plus de lettre, plus de littérature) »; " cette lettre, cette archive qui n'intéresse personne en fin de compte, ni toi ni moi ni personne ${ }^{33}$; etc.

Que faut-il penser de cet " appel au feu » qui rappelle, à plus d'un égard, les procédés de la théologie négative? À titre d'hypothèse, j'avance ceci : la rhétorique de l'échec réinjecte de la distance — une

30. La carte postale, p. 264.

31. Ibid., p. 76.

32. F. KAFKA, Lettres à Milena, pp. 27 ; $91 ; 105 ; 261$.

33. La carte postale, p. $11 ; 35 ; 133$. 
distance nécessaire, qui préserve l'altérité de l'autre et qui l'appelle d'une autre façon, en demandant son appel : celui qui écrit la lettre d'amour veut à son tour être séduit. En se niant, la lettre d'amour demande à être rassurée en s'assurant que l'autre, si elle le désire, va prendre le relais. La tragédie de l'écriture ( "le malheur de la destination ") est aussi ce qui donne à l'écriture sa chance " m'éloigner pour t'écrire ${ }^{34}$ — en donnant à l'autre sa chance, en passant la main. Le don (de l'écriture) se laisse ainsi comprendre, d'une certaine façon, comme une manière de se protéger (de l'autre) : je m'éloigne (en appelant l'autre) pour éviter d'être éloigné (par l'autre $)^{35}$, ou encore je m'éloigne afin de retirer la promesse d'amour que je risque de ne pas pouvoir tenir.

"Ce n'est pas toi que j'aime, c'est bien plus, c'est mon existence, qui m'est donnée à travers toi. »

F. Kafka, Lettres à Milena ${ }^{36}$

La machination de la séduction, on le voit, a plus d'un ressort. Mais s'il faut séduire, nous devons aussi demander à qui profite, en dernière instance, cette séduction. La séduction est-elle totalement indépendante d'un calcul visant à tirer profit de l'autre, contre ou malgré l'autre, en tout cas pour soi? La lettre d'amour relève-t-elle de l'ordre économique ou de l'anéconomie du don? Comment s'écritelle, suivant quelles modalités et à partir de quel lieu? Quel est le rapport de la lettre d'amour au calcul, au système de l'échange - de la dette et du don comme retour (ou comme attente d'un contre-don)? Plus radicalement peut-être, demandons : l'amour qui s'écrit se laisse$\mathrm{t}$-il même penser? Et en se pensant ne se manque-t-il pas toujours, n'est-il pas un amour manqué?

Penser l'amour, c'est le « dialectiser »; c'est l'appréhender comme " mouvement extrême, au-delà de soi, d'un être s'achevant. " ${ }^{37} \mathrm{Un}$ tel

34. Ibid., p. 33.

35. "Je crois que je préfère t'écrire (même quand tu es en face de moi, ou, comme en ce moment à ton secrétaire, tout à côté), tu m'éloignes moins. ", ibid., p. 188.

36. F. KAFKA, Lettres à Milena, pp. 101-102.

37. J.-L. NANCY, Une pensée finie, Paris, Galilée, 1990, (Coll. "La philosophie en effet »), p. 231. 
amour sollicite l'autre, mais en vue d'une fin particulière : l'accomplissement de soi. Dans un tel schème, la lettre d'amour consiste dans l'écriture d'un manque en tant qu'il se comble ou aspire à se combler ; s'élançant vers l'autre mais en vue de soi, la lettre d'amour - ainsi comprise - fait toujours retour, sans s'engager réellement dans les hasards de la destination. L'envoi de la lettre a un sens pour autant qu'elle fournit l'occasion à l'ego de se réfléchir dans la figure d'un alter ego - réduit ici à une figure purement spéculaire, un autre soi, c'est-à-dire tout sauf de l'autre.

L'amour prend la forme de l'amour-propre. Non pas simplement l'amour de soi : l'amour de soi en tant qu'amour de la propriété, l'amour qui travaille à son propre intérêt — bref, l'amour qui se mesure à des valeurs économiques. Même s'il s'écrit, un tel amour ne s'envoie pas. S'il s'envoie, il n'arrive à rien (ni à personne), il revient : "La "lettre" quand tu la prends sur toi [...] n'arrive pas à s'arriver à l'autre. C'est la tragédie de moi dans l'“introjection" : il faut s'aimer pour s'aimer, enfin, si tu préfères, mon amour, pour aimer. ${ }^{38}$

Lorsqu'il s'écrit suivant ces modalités - et peut-il s'écrire autrement?, voilà en somme la question -, l'amour (ou ce qui emprunte ce nom) prend cette forme particulière de "perversion » qui consiste $\grave{a}$ jouir de l'autre. Dans un des récits de La Carte postale, Derrida métaphorise une structure possible de cette « jouissance de l'autre » :

Au dessert, presque sans transition, elle m'a dit qu'elle ne pouvait jouir qu'avec quelqu'un d'autre. Je n'ai pas tout de suite compris la syntaxe de sa phrase. - Mais si, il faut que ce soit quelqu'un d'autre! Et elle éclate de rire en comprenant ce que je ne comprenais pas. Alors elle m'explique ce qu'elle ressentait comme une délicieuse pathologie dont elle n'était pas sûre de pouvoir, en vérité de vouloir guérir : tout se mettait en scène, depuis le début, pour qu'au dernier moment elle pense, imagine, convoque, comment dire, se rende présent un autre que l'autre qui se trouvait à l'instant jouir en elle. Elle ne savait pas si elle faisait exprès mais elle vivait comme une fatalité la nécessité de destiner sa jouissance à l'absent, qui d'ailleurs n'était pas toujours le même, l'autre de l'autre pouvant toujours être encore un autre. C'est naturellement, là il faut que je la cite, un "surcroît » de jouissance toujours disponible, et une " privation mortelle ». Après un silence : le jour où j'aimerai quel-

38. La carte postale, p. 210. 
qu'un, homme ou femme, je suis sûre, enfin je crois que cela cessera, en tout cas je reconnaîtrai l'amour à cela. J'ai beaucoup aimé, pourtant, sans jamais m'abandonner assez à ceux que j'aimais, présentement, je veux dire. Et jusqu'à présent. Autre silence (j'avais déjà demandé l'addition) et sans rien de provocant ni de vulgaire, avec une sorte de confiance à laquelle j'aime encore penser : j'ai le pressentiment qu'avec vous ce serait différent. ${ }^{39}$

Que faut-il penser du rapport à l'autre qui se définit ainsi, entre le " surcroît de jouissance » et la " privation mortelle ", dans le jeu qui va de l'un à l'autre, sans s'interrompre? Suivant le récit de Derrida, il y a bien là une " pathologie » — qualifiée étrangement de « délicieuse ».

Dans la mesure où le pathologique « indique un état morbide, un dérèglement de la finalité vitale par rapport à la santé ", ${ }^{40}$ le problème que pose "la jouissance de l'autre " en est un de vie ou de mort. L'enjeu vital du jouir concerne le soi mais aussi l'autre, car l'expression "jouissance de l'autre " se laisse entendre de deux façons : d'abord comme la jouissance de soi ("...elle vivait comme une fatalité la nécessité de destiner sa jouissance à l'absent...»); mais aussi comme la jouissance de l'autre donnée par le soi ( « l'autre qui se trouvait à l'instant jouir en elle... »).

Dans le premier cas (le jouissance de soi), il faudrait imaginer une lettre d'amour se destinant à elle-même, c'est-à-dire une lettre envoyée à l'autre mais consacrée à la célébration de l'amour de soi — la destination n'étant ici qu'un détour, une stratégie, une façon de retourner à soi (donc au même), une façon de s'économiser. Une telle lettre, on le devine, ne peut que manquer l'autre (comme autre) - n'étant qu'une manière de jouir de soi (par l'autre mais sans l'autre). La lettre d'amour qui se refuse ainsi à se destiner (à l'autre) ne manque pas seulement l'autre, elle manque de l'autre, elle s'en trouve privée. La voix de l'autre inventée par le soi ne peut être que l'écho mortifère de sa solitude : c'est la voix du dernier homme dont parle Nietzsche. ${ }^{41}$

39. Ibid., pp. 67-68.

40. L.-M. MORFAUX, Vocabulaire de la philosophie et des sciences humaines, Paris, Armand Colin, 1980, p. 262. Nous soulignons.

41. "Le dernier philosophe, c'est ainsi que je me nomme, car je suis le dernier homme. Personne ne me parle que moi seul et ma voix me parvient comme celle d'un mourant! Avec toi, voix aimée, avec toi, dernier souffle du 
Dans le second cas (la jouissance de l'autre donnée par le soi), il faudrait imaginer une lettre d'amour arrivant toujours, structurellement, à la mauvaise adresse - la bonne adresse étant toujours une autre adresse. Si l'autre jouit dans le soi, il y a néanmoins méprise car le soi n'est pas vraiment là : il n'est pas là entièrement ou encore pas pour longtemps. À cet égard, chaque mot du récit de Derrida est important. On y lit en effet que l'autre « se trouvait » « à l'instant » jouir en elle : d'une part, l'autre ne jouit pas mais se trouve à jouir — expression qui renvoie davantage aux hasards de la destination qu'à un choix amoureux ; d'autre part, l'autre jouit à l'instant - manière de souligner la précarité du lien et la possibilité imminente de sa fracture.

Dans cette structure donjuanesque, l'autre ne jouit qu'en passant; il n'est connu et reconnu qu'un instant — d'une certaine façon, il n'est pas vraiment reconnu dans sa densité propre. Plus précisément peutêtre, l'autre n'est pas rencontré, puisqu'il s'inscrit simplement comme repère dans un espace provisoire, ouvert puis aussitôt refermé par le désir du soi. L'autre n'est qu'une étape présidant à la construction du soi : la lettre d'amour peut faire jouir sa destinataire accidentelle, c'est le destinateur qui prend le plus de plaisir à cette multiplication des (mauvaises) adresses. Le désir qui se nourrit du changement - du supplément d'altérité — tend à faire de l'autre une victime : l'amourconquête laisse derrière lui des coeurs décimés. ${ }^{42}$

souvenir de tout bonheur humain, laisse-moi encore ce commerce d'une seule heure ; grâce à toi je donne le change à ma solitude et je pénètre dans le mensonge d'une multitude et d'un amour, car mon coeur répugne à croire que l'amour est mort, il ne supporte pas le frisson de la plus solitaire des solitudes et il m'oblige à parler comme si j'étais deux. T'entends-je encore, ma voix? Tu chuchotes en maugréant? Et dût ta malédiction faire crever les entrailles de ce monde! Mais il vit encore et ne me fixe qu'avec plus d'éclat et de froideur de ses étoiles impitoyables, il vit, aussi stupide et aveugle qu'il fût jamais, et $u n$ seul meurt, l'homme. Et pourtant! je t'entends encore, voix aimée! Il meurt encore quelqu'un en dehors de moi, le dernier homme, dans cet univers : le dernier soupir, ton soupir meurt avec moi... », F. NIETZSCHE, Le livre $d u$ philosophe, trad. A. Kremer-Marietti, Paris, Flammarion, (Coll. « GF », 660), 42. On rappelle à cet égard la description très " militaire " de l'amour que Molière met dans la bouche de son Dom Juan : "Les inclinations naissantes, après tout, ont des charmes inexplicables, et tout le plaisir de l'amour est dans le changement. On goûte une douceur extrême à réduire, par cent hommages, 
Par un certain retour de justice, l'ouverture infinie du désir entraîne également dans sa chute le signataire d'une telle lettre d'amour : le donjuanisme implique non seulement la destruction de l'autre mais encore une certaine dissolution du soi. J'évoque ici, sans pouvoir m'y attarder, ce qu'affirme Kierkegaard de «la musicalité absolue de Dom Juan » - qui fait de lui un être "sans existence propre », se hâtant "dans un perpétuel évanouissement ", "justement comme la musique, au sujet de laquelle on peut dire qu'elle est finie dès qu'elle a cessé de vibrer et ne renaît qu'au moment où elle recommence à vibrer. ${ }^{43}$

Ainsi, la pathologie propre à la «jouissance de l'autre » semble liée, du moins en partie, à l'impossibilité de déterminer un partage strict entre ce qui tient du soi et ce qui tient de l'autre. Aimer... T'aimer... S'aimer... Il n'est pas facile de se dégager des entrelacs de l'amour-propre, de sortir de soi. Peut-on aller vraiment ailleurs et rencontrer l'autre comme autre, sur le terrain de l'autre et non plus seulement dans le cercle économique du soi? Peut-on sortir de cette économie? Comment? Cette sortie est-elle vraiment possible? ${ }^{44}$

le coeur d'une jeune beauté, à voir de jour en jour les petits progrès qu'on y fait, à combattre par des transports, par des larmes et des soupirs, l'innocente pudeur d'une âme, qui a peine à rendre les armes, à forcer pied à pied toutes les petites résistances qu'elle nous oppose, à vaincre les scrupules dont elle se fait un honneur et la mener doucement où nous avons envie de la faire venir. Mais lorsqu'on en est maître une fois, il n'y a plus rien à dire ni rien à souhaiter ; tout le beau de la passion est fini, et nous nous endormons dans la tranquilité d'un tel amour, si quelque objet nouveau ne vient réveiller nos désirs, et présener à notre coeur les charmes attrayants d'une conquête à faire. ", MOLIÈRE, Dom Juan, Paris, EJL, (Coll. "Librio », 14), 1995, acte I, scène II (p. 16-17).

43. S. KIERKEGAARD, "Les étapes érotiques spontanées ou l'érotisme musical », dans Ou bien... Ou bien..., trad. F. et O. Prior et M.H. Guignot, Paris, Gallimard, 1943, p. 81.

44. Il faudrait demander aussi: la sortie de l'économie est-elle même souhaitable? Après tout, si Derrida souligne le caractère pathologique de la " jouissance de l'autre ", il qualifie néanmoins cette pathologie de " délicieuse ", suggérant par là qu'elle n'appelle peut-être pas une thérapeutique (" elle m'explique ce qu'elle ressentait comme une délicieuse pathologie dont elle n'était pas sûre de pouvoir, en vérité de vouloir guérir»). Il n'est pas facile de renoncer au " "surcroît" de jouissance toujours disponible » que procure 
"C'est une lettre qui dit tout mais dont le contenu n'est jamais révélé, car elle est donnée une fois pour toutes. »

D. Wills, «Donner à la lettre » 45

Le texte derridien nous conduit à une zone grise. Non seulement la destination de la lettre d'amour paraît incertaine mais le geste qui motive son écriture et son envoi semble équivoque. D'une part. en tant que réponse, la lettre d'amour se situe d'emblée dans le système économique de l'échange, sous la forme particulière du " remboursement de dette » : la lettre d'amour comme envoi d'un disciple à son maître. ${ }^{46} \mathrm{D}$ 'autre part, en tant qu'inscription inauguratrice de mémoire, la lettre d'amour tend à instituer un rapport d'échange qui s'avère incompatible avec le caractère résolument " oublieux » du don amoureux : "Pas de don sans oubli absolu (qui t'absolve aussi du don, et de la dose), oubli de ce que tu donnes, à qui, pourquoi et comment, de ce que tu t'en rappelles ou espères. Un don, s'il y en a, ne se destine plus. ${ }^{47}$

la « jouissance de l'autre ». À cet égard, Derrida se démarque nettement de la résolution de Kant en faveur de l'amour moral du prochain (effet d'une volonté désintéressée) et de sa déconstruction du sentiment amoureux — qualifié par Kant de "pathologique » sans rapport à quelque forme de délice que ce soit ; voir E. KANT, "Des mobiles de la raison pure pratique ", dans Critique de la raison pratique, trad. F. Picavet, Paris, Presses Universitaires de France, (Coll. "Bibliothèque de philosophie contemporaine »), 1976, pp. 75-94.

45. D. WILLS, «Donner à la lettre ", dans J.-M. RABATÉ et M. WETZEL (dir.), L'éthique du don : Jacques Derrida et la pensée du don, Paris, MétailiéTransition, 1992, p. 160.

46. " Pour me rendre avec toi au-delà du principe de paiement (c'est le seul pas que j'aime, le seul qui m'intéresse), je dois te parler interminablement de dette, et d'argent, de sacrifice, d'ingratitude (le mienne est démesurée à ton égard), de culpabilité et d'acquittement, de vengeance sublime et de comptes à régler. Je dois t'en parler. Je te dois d'en parler. Je serai toujours demandeur avec toi. Notre alliance fut aussi cette économie domestique. ", La carte postale, p. 252.

47. Ibid., p. 181. Dans une lettre adressée à l'évêque Mynster, Kierkegaard évoque quelques modalités de cet oubli nécessaire du don : «Il est certain que, pour celui qui offre un don, il n'existe dans le fait de donner ou d'avoir donné 
Qu'elle soit instauratrice ou qu'elle s'inscrive comme réponse dans le régime économique, la lettre d'amour reste une affaire de dette et de remboursement, de culpabilité et d'ingratitude - chaque envoi épistolaire répète la grande scène d'Éden. Pourtant, Derrida envisage la rupture possible du cercle économique : "Le jour où j'aimerai quelqu'un, homme ou femme, je suis sûre, enfin je crois que cela cessera, en tout cas je reconnaîtrai l'amour à cela. » Il faut souligner l'hésitation qui creuse la phrase, substituant un rapport de foi (« je crois... ») à un rapport de certitude (« je suis sûre... »). Cette hésitation en dit déjà beaucoup sur le nature du rapport amoureux : un rapport fragile, susceptible d'être rompu à tout moment et de retomber, par là, dans l'ordre économique du calcul.

Derrida envisage d'autres modalités de rupture du cercle économique. On peut évidemment envisager l'éviction de l'un des termes du rapport : la suppression de soi étant évidemment la solution la plus "facile ». ${ }^{48}$ La lettre d'amour peut aussi se dérober à l'échange - et à l'ordre qui s'organise autour d'elle — en se supprimant elle-même. Ce travail de mise en abîme n'est pas étranger en tout cas à ce qui se dessine dans La carte postale.

À travers la détermination d'un rapport complexe à la temporalité - rapport qui demanderait lui-même à être analysé en détails - , les « Envois » de Derrida s'ouvrent par la formulation d'un regret : "Il aurait fallu tout brûler. " ${ }^{49}$ Et en écho à ce voeu et en guise de conclusion inclusive, Derrida écrit lors du dernier envoi :

(et ce, quelque bienvenu qu'ait été le don pour le destinataire, et quel que soit l'empressement de celui-ci à remercier), aucune obligation de faire le prochain pas, un pas qui serait une nouvelle faveur de la part du donateur - ou peutêtre est-ce la même, répétée une fois encore : accepter les remerciements du destinataire. Alors je vous prie de me permettre de remercier ; ou plutôt, puisque je ne peux entendre votre réponse, je vous prie de me pardonner de vous gratifier, en guise de remerciement pour le cadeau reçu, presque d'une ingratitude, dans la mesure où cette petite lettre vous dérange encore en vous faisant perdre un instant. ", S. KIERKEGAARD, " Lettres choisies sur l'amour et le don ", trad. A.-C. Habbard, Philosophie 60 (1998), pp. 19-20. 48. Le motif du suicide est un thème obsédant au début de La carte postale (p. 19 et suivantes).

49. Ibid., p. 11. 
Demain je t'écrirai encore, dans notre langue étrangère. Je n'en retiendrai pas un mot et en septembre, sans que je l'aie même revue, tu brûleras tu la brûleras, toi, faut que ce soit toi. ${ }^{50}$

Rapportant la structure qui soutient La carte postale à la lettre amour, il faudrait lire celle-ci dans l'entre-deux de sa propre mort. L'ambiguïté mais aussi la profondeur de l'écriture amoureuse - et peut-être aussi de toute écriture — résiderait dans la conjonction de la fragilité la plus extrême (la fragilité de celui qui se sait mortel) et de la force la plus puissante. Car la lettre d'amour témoigne aussi d'une force, d'un pouvoir réel : l'amour en s'écrivant défie d'une certaine façon les limites du possible, les limites de la mort. Envoyer une lettre, c'est affirmer que l'indicible peut et doit encore être dit.

L'ineffable dont on nous rebattra les oreilles n'est qu'un alibi. Ou signe de paresse. On peut toujours tout dire, le langage contient tout. On peut dire l'amour le plus fou, la plus terrible cruauté. On peut nommer le mal, son goût de pavot, ses bonheurs délétères. On peut dire Dieu et ce n'est pas peu dire. On peut dire la rose et la rosée, l'espace d'un matin. On peut dire la tendresse, l'océan tutélaire de la bonté. On peut dire l'avenir, les poètes s'y aventurent les yeux fermés, la bouche fertile. On peut tout dire de cette expérience. Il suffit d'y penser. Et de s'y mettre. D'avoir le temps, sans doute, et le courage, d'un récit illimité, probablement interminable, illuminé - clôturé aussi, bien entendu — par cette possibilité de se poursuivre à l'infini. Quitte à tomber dans la répétition et le ressassement. Quitte à ne pas s'en sortir, à prolonger la mort, le cas échéant, à la faire revivre sans cesse dans les plis et les replis du récit, à n'être plus que le langage de cette mort, à vivre à ses dépens, mortellement. ${ }^{51}$

Ainsi l'indicible peut être dit, mais jamais complètement. Si le signataire de la lettre d'amour demande la " consumation postale » c'est qu'il sait que la lettre ne peut que faillir ; par ailleurs, s'il l'écrit quand même, malgré tout, c'est pour le reste, l'après-cendre. Qu'estce qui survivra au feu? Le geste d'écriture lui-même. Tout ce qui sera dit dans la lettre d'amour ne compte pas ; ce qui compte tient au rapport lui-même. (Le Banquet de Platon revêt ici un caractère paradigmatique dans la mesure où s'y opère la mise en scène de cette

50. Ibid., p. 273.

51. J. SEMPRUN, L'écriture ou la vie, Paris, Gallimard, 1994, (Coll. « Folio ", 2870), p. 26. 
confusion fondamentale : confusion entre l'amour de ce qui est dit la chose, le contenu - et l'amour de celui qui la dit la relation instituée par le geste de l'autre. En confondant l'amour de la sagesse et l'amour du maître, Alcibiade illustre un schème qui ne cessera jamais d'être reproduit.)

En tant que geste, la lettre d'amour n'est pas sans ressembler à la prière - entendue non comme "l'acte subjectif d'un coeur sensible " mais comme la figure qui «signe le parcours de la distance ${ }^{52}$. Comme la prière, la lettre d'amour tend à se réduire à l'adresse, à l'interpellation : "Je ne t'écris plus rien d'autre, je t'écris seulement, toi, à toi. " ${ }^{53}$ Par-delà tout contenu, en dehors de toute référence à ce qui est dit ou écrit, la lettre d'amour s'adresse à l'autre; comme la prière qui « ne parle pas de mais $\grave{a} » .{ }^{54}$ Cette adresse, certes, ne garantit en rien l'instauration d'un rapport mais, d'une certaine façon, elle lui donne déjà sa chance, elle en ouvre la possibilité. Cela suffit peutêtre.

On comprend dès lors que l'écriture amoureuse du « viens! » évoquée précédemment - est irréductible au registre impératif : l' « ordre » qui s'y donne à entendre relève au contraire de « la prière la plus abandonnée ${ }^{55}$, celle qui appelle l'autre, qui lui demande sans cesse de s'inventer lui-même, à chaque instant. En tant que prière, la lettre d'amour préserve l'altérité de l'autre, en ne la convoquant pas mais en l'invoquant, en espérant simplement qu'elle se reconnaisse, c'est-à-dire en espérant rien, en s'adressant à elle, à Lui.

C'était comme une lettre, une lettre d'amour. De moi à toi. Sans espoir de correspondance, sans attente de retour. Comme une vraie lettre, comme un envoi qui visait l'autre (en l'occurrence toi, car c'est à toi qu'elle s'adressait), qui s'y destinait mais sans espérer un quelconque retour (de courrier). Presque sans attendre donc. En tout cas, certainement sans y

52. J.-L. MARION, L'idole et la distance, Paris, Grasset, (Coll. « Le livre de poche/Biblio-essais ", 4073), 1977,p. 198.

53. La carte postale, p. 182.

54. J. DERRIDA, "Comment ne pas parler : dénégations », dans Psyché, p. 574.

55. La carte postale, p. 66. 
compter; on le sait maintenant, ou plutôt on l'espère, une lettre d'amour échappe au calcul.

Il ne faut pas s'y tromper, en dépit des apparences, on ne s'est pas écarté - je ne pouvais pas m'écarter - de la lettre, donc d'un certain ton, d'un certain genre, d'un certain " je " et d'un certain " $t u$ " c'est à toi qu'elle s'adressait cette lettre, et elle était de moi. Ma signature mon dernier mot, sans doute.

- Tu vois! Ta réponse, je ne l'attends déjà presque plus. Le dernier mot ne viendra pas de toi, il me reviendra (sans être passé par toi).

On ne s'est donc pas écarté - je ne pouvais pas m'écarter — de la lettre, de ce qu'elle implique, de ce qu'elle appelle, de ce qui s'y joue. Mais c'était une lettre cachée; il te fallait lire entre les lignes, entre les mots. Ce n'était pas une lettre. Ou plutôt, ça ne se donnait pas comme une lettre. Une politique - concertée, délibérée, affirmée - du secret, voilà ce qu'il fallait mettre en oeuvre. Ça ne se disait pas. Ça ne disait pas "je ", ça ne disait pas "tu", même si c'est à toi - je le répète encore - que la lettre s'adressait, même si elle était de moi: de moi à toi.

Il fallait solliciter ici le discours de l'objectivité, se cacher (toi et moi) derrière un "nous "; il fallait renoncer à se dire, à dire "je ", à dire «tu». Pas complètement pourtant, car le biais choisi- ma stratégie! - était loin d'être étranger au genre épistolaire. En proposant une lecture de "La carte postale» de Jacques Derrida, je choisissais de commenter un discours lié inextricablement à une "poétique de l'apostrophe"; un discours s'inscrivant d'emblée dans l'espace ouvert par l'appel de l'un à l'autre, d'un moi à un toi : "Le mot - apostrophe -, il dit la parole adressée à l'unique, l'interpellation vive (l'homme de discours ou d'écriture interrompt l'enchainement continu de la séquen$c e$, d'une volte il se trourne vers quelqu'un, voire quelque chose, il s'adresse à toi). " ${ }^{56}$

Ainsi je pouvais continuer à dire "je " mais en me cachant dans le "je» d'un autre, en m'y logeant subrepticement, sans qu'on y prenne bien garde. Derrida était l'homme entre nous - étrange perversion sexuelle jouant la relation binaire sous un mode ternaire. Comme dans toutes les vraies histoires d'amour, nous étions plus que deux. Mais tu le sais maintenant, j'étais là ; quand il parlait, c'était moi.

56. Ibid., p. 8 . 
La lettre que tu as lue était tachée de mon sang et de mes pleurs; ainsi la méprise de Roxane n'a pas été répétée - j'ai été tout à la fois Cyrano et Christian.

Voilà! C'était ma lettre d'amour, une lettre impossible ; je te l'ai envoyée, je te l'envoie encore, sans espoir qu'elle te parvienne...

\section{RÉSUMÉ}

Rapporter le geste théologique à la lettre d'amour, c'est s'inscrire d'entrée de jeu dans un espace assez inconfortable. C'est s'engager à s'exprimer en exilé dans l'interstice formé par un double écart : d'une part, l'écart qui sépare l'écriture théologique de l'écriture amoureuse et, d'autre part, l'écart qui éloigne chacune de ces écritures de son lieu de circulation habituel. Loin de vouloir résorber ces écarts, je voudrais m'y enfoncer comme il faut, en étendre les ressorts afin de mesurer le jeu qu'ils permettent. Pour ce faire, je propose de prendre comme fil conducteur La carte postale de Jacques Derrida et l'horizon de questionnement que ce livre étrange délimite.

\section{ABSTRACT}

From the outset, the work of transposing the theological movement to a love letter is an uncomfortable one. It engages us to express ourselves in exil, in a space formed by a double gap ; on one side a gap that separates theological writing from loving writing, and on the other side a gap that pushes away both writings from their usual surroundings. Far from wanting to absorb these gaps, I would like to drive right into it, to spread their elasticity in order to measure the extension of their movement. To do so, I propose to use La carte postale from Jacques Derrida as a conductor thread and for the horizon of questioning raised by it. 